\title{
Methodology for the Synthesis of Methacrylate Monomers Using Designed Single Mode Microwave Applicators
}

Received 00th January 20xx, Accepted 00th January 20xx

\author{
Adam A. Dundas ${ }^{\mathrm{a}, \mathrm{b}}$, Andrew L. Hook ${ }^{\mathrm{b}}$, Morgan R. Alexander ${ }^{\mathrm{b}}$, Samuel W. Kingman ${ }^{\mathrm{a}}$, Georgios \\ Dimitrakis*a and Derek J. Irvine*a
}

DOI: $10.1039 / x 0 x \times 00000 x$

\begin{abstract}
A novel single-well prototype high throughput microwave reactor geometry has been produced and shown to be capable of synthesizing an array of non-commercially available methacrylate monomers. The reactor, which delivers the energy required via a dedicated coaxial line, has been shown experimentally to outperform other conventional/microwave formats. It is demonstrated to achieve significantly higher conversions than the alternative reactor types, whilst requiring (a) low levels of input power, (b) no additional energy for agitation/mass transfer, (c) no solvent and (d) no environmentally impacting thermos-fluids.
\end{abstract}

In recent years, high throughput materials discovery (HTMD) programs have accelerated the identification of novel materials ideally suited for target applications. Such HTMD campaigns often make use of platform technologies such as polymer microarrays. ${ }^{1-3}$ Consequently, microarrays have enabled thousands of materials to be screened in a single experiment, allowing for novel biomaterials to be developed for applications such as reducing bacterial attachment to medical devices, the expansion of pluripotent stem cells, increasing the maturity of cardiomyocytes derived from stem cells and providing bio instructive implant materials. ${ }^{4-9}$

The adoption of such HTMD techniques can radically change the sustainability profile of such extended research programs targeted at identifying maps the links between molecular structure and application performance. The use of polymer microarrays for initial activity assessments means that very small quantities (i.e. microliters) of feedstock are used and one researcher can complete a series of up to 350 reagent mixture evaluations in triplicate in less than a day. ${ }^{6}$ To conduct the same number of synthetic preparations in conventional glassware would take months/years of manual effort and multiple

\footnotetext{
a. Department of Chemical and Environmental Engineering, Faculty of Engineering, University of Nottingham, NG7 2RD

b. Advanced Materials and Healthcare Technologies, School of Pharmacy, University of Nottingham, NG7 2RD

*. Corresponding authors

Electronic Supplementary Information (ESI) available: [details of any supplementary information available should be included here]. See DOI: 10.1039/x0xx00000x
}

kilograms of feedstock. Rather, these HTMD strategies allow the conventional labour and reagent intensive work to be focused down onto a very few target combinations. Reducing waste in terms of reagents, solvents, energy, and researcher time needed to identify the key data sought.

Typically, due to the large amounts of different materials required to build the large data sets required to draw strong conclusions from a HTMD study, readily accessible commercially available chemicals are utilized to create a material library. ${ }^{10}$ However, this leads to two issues; a) commercial materials often have limited chemical diversity thus a vast majority of chemistries are left unexplored, b) an inability to probe the effect of finite changes in chemical moieties on specific structure-activity relationships. Thus, synthesizing specific monomers for HTMD programs designed using the predictive models built on data from assessing commercial moieties can produce a monomer library predicted to be fit for purpose. The continued refinement of molecular structures is vital to optimizing the performance of subsequent biomedical devices.

Therefore, an area where the sustainability of HTMD programs can be improved is by the development of more efficient, multiwell library apparatus that allow reliable rapid throughput synthesis (RTPS) of novel monomers. Many of the RTPS systems marketed to-date involve designs that are based on combinations of multiple, conventional thermo-fluid heated and agitated reactors, which have not included any consideration of minimizing environmental impact, carbon footprint or chemical waste. ${ }^{11}$ Microwave heating (MWH) has been shown in literature to increase reaction rates with lower power inputs (i.e. 10's of Watts in total) compared to conventional heating methods. ${ }^{12,13}$ Thus $\mathrm{MWH}$ has significant potential to remove the need to use highly environmentally impacting thermo-fluids and reduce RTPS energy usage. The volumetric nature of $\mathrm{MWH}$ has also been shown to deliver greater control over reaction medium temperature, so reducing/removing the need to input additional energy to agitate the contents to overcome any thermal gradients in the wells. ${ }^{12}$ Solvent free chemistry has also been extensively reported using microwaves. ${ }^{14}$ 
The work herein describes the development and use of an individual microwave heated "well" reactor ("micro-well") for a target, solvent-free transesterification reaction. It will ultimately form the basis of a multi-well microwave synthesis system, which involves minimal energy and waste and a schematic can be found in Figure S1.

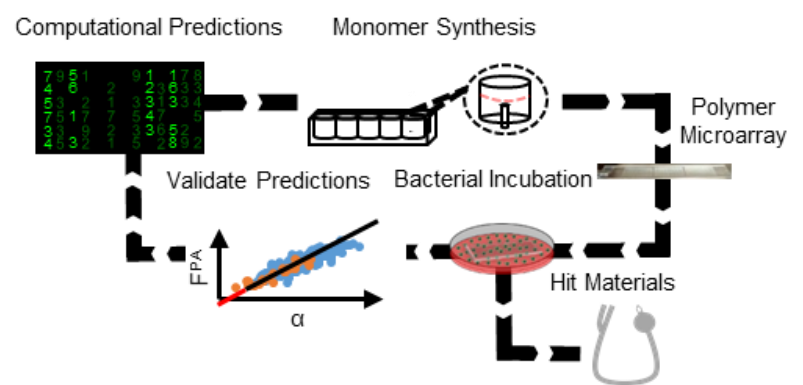

Figure 1. Schematic showing proposed materials discovery feedback loop, where predicted materials are synthesised using the RTPS microwave synthesis reactor

Computational simulations led to the construction of a working single micro-well applicator capable of reaching $160{ }^{\circ} \mathrm{C}$ with only $40 \mathrm{~W}$ of applied power. The wide utility of this system was demonstrated by the synthesis of a range of novel methacrylate monomers.

A commercially available electromagnetic simulation software Concerto ${ }^{\circledR}$, supplied by Vector Fields, was used to simulate the microwave field pattern for the safe design of a micro-well capable of conducting the target (trans)esterification mechanism using methanol as a model alcohol (Figure 2).
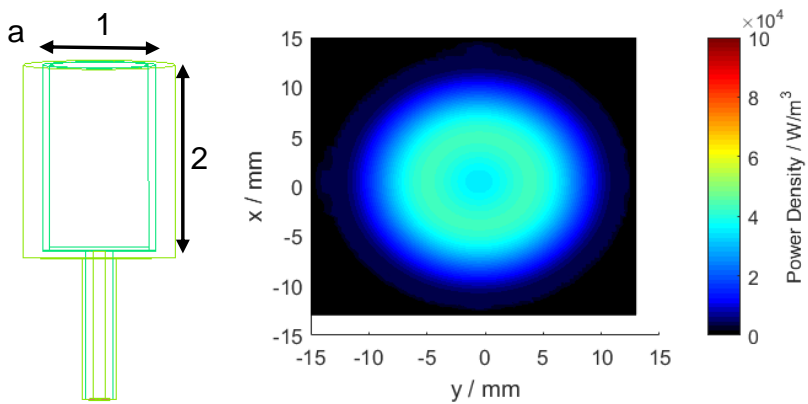

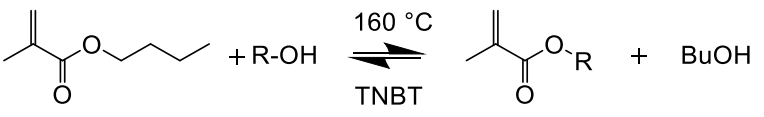

Figure 2. Solvent free transesterification method used to synthesize novel methacrylates in the micro-well at $160{ }^{\circ} \mathrm{C}$ with titanium butoxide (TnBT) as catalyst.

The electromagnetic study of the geometrical design of the micro-well used finite-difference time domain (FDTD) simulations. ${ }^{16,17}$ Such FDTD simulations have been used to simulate a range of antennas for different applications, including medical imaging and selective heating of tumors. ${ }^{18,19}$ These simulations were used to optimize well radius, antenna length and shape (see supplementary information) to achieve the most uniform filed density throughout the medium. Figure 3 shows the power density distribution when the reactor is filled with methanol. Methanol was selected because it represents a worst case scenario in terms of its dielectrics properties and thus penetration depth of the electromagnetic energy throughout the bulk. Therefore, during our chemical synthesis experiments, we expect a power density uniformity which is equivalent to that depicted in Figure 3. Consequently, we can infer that the expected temperature distribution, allowing for heat losses, will also be similar.

The cavity designs in Figure 3 were shown to operate below the cut-off frequency to prevent microwave leakage at a frequency of $2.45 \mathrm{GHz}$. It was shown during a computer simulation that for the optimum design (Figure $3 b$ ), only $\sim 0.1 \mu \mathrm{W} / \mathrm{cm}^{2}$ was

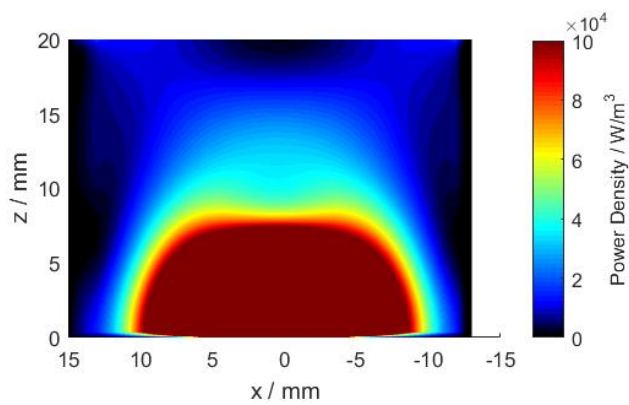

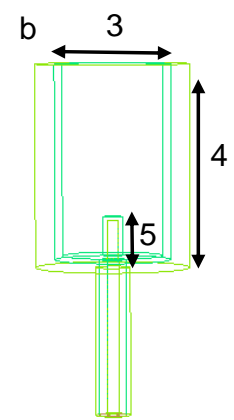
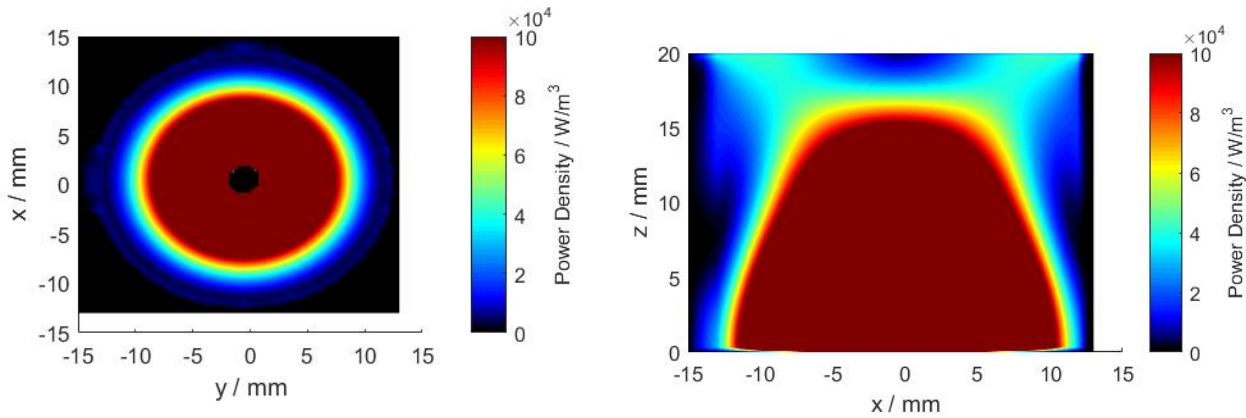

Figure 3. Schematic of the internal reactor structure and computational simulations showing the power desity distribution when the reactor is filled with methanol via the open top prior ot the evaluation at $2.45 \mathrm{GHz}$ for a) no antenna and b) cylindrical antenna. Well geometries are 1) $30 \mathrm{~mm}$ diameter, 2) $50 \mathrm{~mm}$ length, 3) $30 \mathrm{~mm}$ diameter 4) $50 \mathrm{~mm}$ length and 5) $10 \mathrm{~mm}$ length. All simulations used a coaxial power of outer diameter $D=7 \mathrm{~mm}$ and internal diameter of $3.5 \mathrm{~mm}$ to deliver power to reactor. Geometries were described using a mesh of 850,000 cells over 30,000 iterations which were applied with $10 \mathrm{~W}$ of power and analyzed at $\mathrm{XY}=10 \mathrm{~mm}$ and $\mathrm{XZ}=$ $2.5 \mathrm{~mm}$ respectively. 
detected outside of the well, where the maximum permissible dosage limit is $5 \mathrm{~mW} / \mathrm{cm}^{2}$ (Figure S6). ${ }^{15}$ This demonstrated that the design was working below the cut-off frequency at 2.45 $\mathrm{GHz}$. Calculating the cut off frequency for the design also allowed us to design a choke which is as short as feasibly possible to allow for a higher efficiency of mass transfer of byproducts, which is an element of design not possible in conventional microwave heating. More detail can be found in supplementary Figure S7.

This also demonstrated the benefit of utilizing a monopole antenna as this format allowed microwaves to couple with the antenna and hence increase penetration into the mixture and also resulted in a more uniform power density field distribution (Figure 3). Similar monopole antennas designs have been shown to be highly effective at manipulating the power density distribution to optimize drilling in ceramic materials. ${ }^{20,21}$ The final optimized micro-well shown is shown in Figure $3 \mathrm{~b}$.

Following simulation studies, a working prototype micro-well was manufactured and linked to a solid-state microwave generator (Miniflow 200SS) via a coaxial tuner, which was used to match the input power into the sample. A feedback loop using a fibre optic cable monitored and controlled the reaction temperature. An alcohol featuring the desired functionality was used with butyl methacrylate (BMA) to develop the novel methacrylate monomers. The transesterification chemistry applied utilized TNBT as a catalyst at a $1 \%$ molar ratio relative to butyl methacrylate. This chemistry (Figure 2) was chosen because it is well understood and industrially applied. Thus, any deficiency in the results would be attributed to the reactor design and the results should be scalable. This allowed a diverse range of monomers to be synthesized as there are many commercial alcohols available where the corresponding (meth)acrylate is not commercially available. The reaction is limited to the use of alcohols with boiling point's similar or above that of the set reaction temperature chosen to prevent excessive loss of alcohol. The optimized Microwell format reactions to synthesize cyclododecyl methacrylate (CyDMA) and 3-phenyl-2-propenyl methacrylate (PpMA) were conducted at $160{ }^{\circ} \mathrm{C}$ whilst for 4 -methyl-2-pentyl methacrylate $140{ }^{\circ} \mathrm{C}$ (MpMA) was used due to 4-methyl-2-pentanol's boiling point $\left(132{ }^{\circ} \mathrm{C}\right)$, with the structures shown in Figure 4.<smiles>C=C(C)C(=O)OC1CCCCCCCCCC1</smiles>

CyDMA

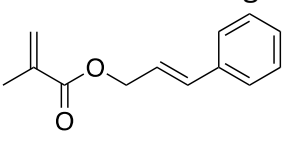

PpMA

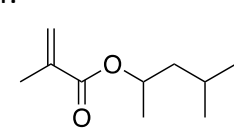

MpMA
Figure 4. Molecular structures of CyDMA, PpMA and MpMA monomers synthesized using TNBT as a catalyst

The conversions achieved were also compared to the same reaction conditions conducted using a standard conventional oil bath and a single mode microwave reactor with waveguide (Sairem Miniflow 200SS) with both methods using identical glassware such that the Micro-well reactor performance could be compared to both standard microwave and conventional heating methods (Table 1). Conversion characterization was conducted using ${ }^{1} \mathrm{H}$ NMR to determine reactor performance. Mass transfer was examined by using ${ }^{1} \mathrm{H}$ NMR to observe residual butanol in the final reaction mixture. Example NMR spectra and conversions can be found in the supplementary information.

Table 1. Transesterification synthesis comparison using conventional, microwave and micro-well prototype methodologies for the target novel monomers. Samples were prepared in a ratio of 7:5 BMA: alcohol with $1 \%$ molar ratio of TNBT relative to BMA and then heated for 150 minutes. Conversions were obtained through ${ }^{1} \mathrm{H}$ NMR. All reaction conversions have been quoted relative to the consumption of alcohol

\begin{tabular}{llll}
\hline Apparatus & \multicolumn{2}{l}{ Conversion (\%) } \\
type & CyDMA & PpMA & MpMA \\
\hline Conventional & 48 & 41 & 45 \\
Microwave & 52 & 15 & 48 \\
Micro-well & 78 & 80 & 73 \\
\hline
\end{tabular}

Table 2. Remaining butanol leftover in transesterification experiments after $150 \mathrm{~min}$. Butanol percentage was calculated relative to the final product.

\begin{tabular}{llll}
\hline Apparatus & \multicolumn{2}{l}{ Remaining Butanol(\%) } \\
type & CyDMA & PpMA & MpMA \\
\hline Conventional & 50 & 47 & 48 \\
Microwave & 44 & 58 & 49 \\
Micro-well & 2 & 3 & 16 \\
\hline
\end{tabular}

Conversions in the conventional and standard microwave methods were largely limited to below 50\% whilst using identical glassware. This was attributed to mass-transfer limitations within the reaction vessel which only have small ports, i.e. an inability to remove the by-product butanol from the reaction mixture influencing the reversibility of the reaction equilibrium (Figure 2). However, the open topped micro-well reactor design shown in Figure $3 \mathrm{~b}$ overcame these masstransfer effects such that it exhibited conversions of $70-80 \%$. This was also demonstrated in Table 2 which showed the large reduction in residual butanol in the final product compared to both conventional and conventional microwave heating methods. The open top vessel could also allow for constant monitoring systems such as heating profiles to aid reaction progression. This showed that the microwell was able to synthesise a range of different structures of monomers including large sterically hindered monomers such as CyDMA and outperformed conventional and microwave heating methods for the transesterification of bespoke methacrylate monomers.

These results showed the utility of this initial micro-well design and its potential to be scaled into a multi-well system based on its "chemical flexibility". Moreover, whilst the track record of up-scaling microwave processes has been poor. This has predominantly related to an inability to increase the reactor volume and retain the microwave heating benefits e.g. problems with energy penetration depth. This case is different 
in that the reactor volume is going to be retained, as should be the microwave benefits. Rather it is the number of individual reactors that will change, so scale up is related to the electrical engineering design of a system to supply energy multiple reactors via coaxial lines. Thus, to test the viability of creating a multi-well system, a series of experiments were performed to test the minimum power input required to achieve desired reaction conditions to simply this electrical engineering design. The reaction to generate CyDMA was heated in the micro-well applying 20, 40 and $60 \mathrm{~W}$ (Figure 5).

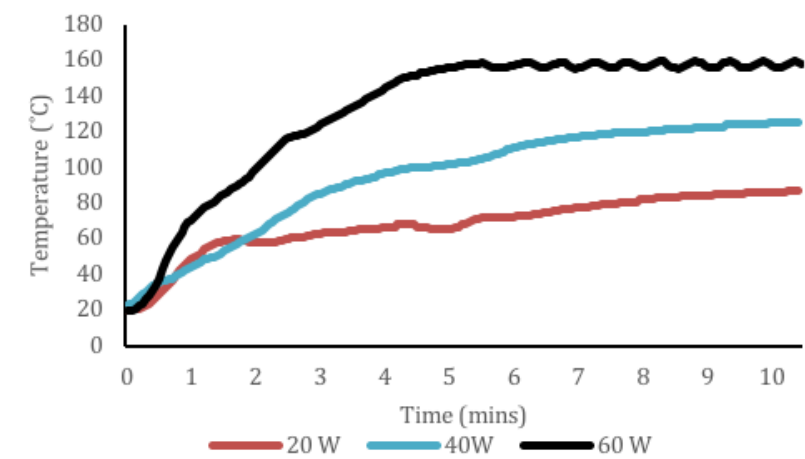

\begin{tabular}{llll}
\hline & $20 \mathrm{~W}$ & $40 \mathrm{~W}$ & $60 \mathrm{~W}$ \\
\hline Time (mins) & N/A & 50 & 5
\end{tabular}

Figure 5. Heating profiles for synthesis of CyDMA using different input powers for a target temperature of $160{ }^{\circ} \mathrm{C}$

$20 \mathrm{~W}$ was shown to be insufficient to heat the reaction mixture to the target of $160{ }^{\circ} \mathrm{C}$, but this target was attained with $40 \mathrm{~W}$ and $60 \mathrm{~W}$ in 50 and 5 minutes respectively. Hence, this data suggests that a 12-well system would require a constant energy demand of $720 \mathrm{~W}$ for the first 5 minutes, followed by lower levels of pulsed microwave power thereafter to maintain reaction temperature. Furthermore, such microwave powers are readily available commercially so the construction of a 12 well system is eminently possible with current equipment.

\section{Conclusions}

We have developed a novel, operational, $15 \mathrm{ml}$ scale, singlewell microwave prototype reactor geometry and demonstrated that is it capable of synthesising new methacrylate monomers. The performance of this bespoke design was shown exhibit a combination of benefits that differentiated its performance from most conventionally applied synthetic methods. It required very low levels of input power (40 - $60 \mathrm{~W}$ ), no additional energy for agitation/increasing mass transfer effects, no solvent and no environmentally impacting thermos-fluids. Therefore, this reactor has shown potential to form the basis of design for developing a low-environmentally impacting, multiple well microwave heated RTPS reactor. The aim is to employ such an apparatus to develop large numbers of novel materials for HTMD programs and so vastly increase the rate at which library candidates can be synthesised. The micro-well reactor was shown to outperform both standard conventional and microwave techniques by overcoming mass transfer effects. It has also been shown the benefits of using monopole antennas to improve microwave penetration, achieve more uniform power density distributions and therefore greater reactor efficiencies.

During this study we have demonstrated the capability of heating a transesterification experiment to $160{ }^{\circ} \mathrm{C}$ with only 40 $\mathrm{W}$ of power to develop a range of novel methacrylate monomers including branched, aromatic and cyclic methacrylate structures. This has proven the feasibility of the apparatus depicted in Figure 1 to be able to produce a RTPS library synthesiser capable of supporting HTMD programs with judiciously chosen libraries.

\section{Acknowledgements}

The authors would like to acknowledge the funding received from the EPSRC DTC studentship programme and the Faculty of Engineering. The authors would also like to thank Dr Ben Muir of the CSIRO's Rapid Automated Materials \& Processing Centre for useful discussions.

\section{Conflicts of interest}

There are no conflicts to declare

\section{Notes and references}

M. S. Algahtani, D. J. Scurr, A. L. Hook, D. G. Anderson, R. S. Langer, J. C. Burley, M. R. Alexander and M. C. Davies, J. Control. Release, 2014, 190, 115-26. A. L. Hook, C.-Y. Chang, J. Yang, S. Atkinson, R. Langer, D. G. Anderson, M. C. Davies, P. Williams and M. R. Alexander, Adv. Mater., 2013, 25, 2542-7.

J. Kohn and T. Road, Nat. Mater., 2004, 3, 745-7.

D. G. Anderson, S. Levenberg and R. Langer, Nat. Biotechnol., 2004, 22, 863-6.

L. K. Hansen, M. Brown, D. Johnson, D. F. Palme, C. Love and R. Darouiche, PACE - Pacing Clin. Electrophysiol., 2009, 32, 898-907.

A. L. Hook, C.-Y. Chang, J. Yang, J. Luckett, A. Cockayne, S. Atkinson, Y. Mei, R. Bayston, D. J. Irvine, R. Langer, D. G. Anderson, P. Williams, M. C. Davies and M. R. Alexander, Nat. Biotechnol., 2012, 30, 868-75. G. Tourniaire, J. Collins, S. Campbell, H. Mizomoto, S Ogawa, J.-F. Thaburet and M. Bradley, Chem. Commun. (Camb)., 2006, 2118-20.

8 A. D. Celiz, J. G. W. Smith, A. K. Patel, A. L. Hook, D. Rajamohan, V. T. George, L. Flatt, M. J. Patel, V. C. Epa, T. Singh, R. Langer, D. G. Anderson, N. D. Allen, D. C. Hay, D. A. Winkler, D. A. Barrett, M. C. Davies, L. E. Young, C. Denning and M. R. Alexander, Adv. Mater., 2015, 27, 40064012.

9 A. K. Patel, M. W. Tibbitt, A. D. Celiz, M. C. Davies, R. 
Langer, C. Denning, M. R. Alexander and D. G. Anderson, Curr. Opin. Solid State Mater. Sci., 2016, 20, 202-211.

A. L. Hook, D. G. Anderson, R. Langer, P. Williams, M. C. Davies and M. R. Alexander, Biomaterials, 2010, 31, 18798.

11 V. Polshettiwar and R. S. Varma, Chem. Soc. Rev., 2008, 37, 1546-1557.

12 C. O. Kappe, Angew. Chem. Int. Ed., 2004, 43, 6250-6284.

13 J. Robinson, S. Kingman, D. Irvine, P. Licence, A. Smith, G. Dimitrakis, D. Obermayer and C. O. Kappe, Phys. Chem. Chem. Phys., 2010, 12, 4750-8.

14 M. B. Gawande, V. D. B. Bonifácio, R. Luque, P. S. Branco and R. S. Varma, ChemSusChem, 2014, 7, 24-44.

15 A. C. Metaxas and R. J. Meredith, in Industrial Microwave Heating, eds. A. T. Johns, G. Ratcliff and J. R. Platts, IET, 2008, pp. 277-295.

16 K. Yee, IEEE Trans. Antennas Propag., 1966, 14, 302-307.

17 J. Robinson, S. Kingman, D. Irvine, P. Licence, A. Smith, G. Dimitrakis, D. Obermayer and C. O. Kappe, Phys. Chem. Chem. Phys., 2010, 12, 10793.

18 D. Ireland and A. Abbosh, IEEE Trans. Antennas Propag., 2013, 61, 2352-2355.

19 B. T. Mcwilliams, E. E. Schnell, S. Curto, T. M. Fahrbach and P. Prakash, IEEE Trans. Biomed. Eng., 2015, 62, 2144-2150.

20 E. Jerby, V. Dikhtyar, O. Aktushev and U. Grosglick, Science (80-. )., 2002, 298, 587-589.

21 E. Jerby, O. Aktushev and V. Dikhtyar, J. Appl. Phys., 2005, 97, 1-7. 